\title{
Duplicidade da veia renal direita em gato-do- mato-pequeno (Leopardus guttulus): relato de caso
}

\author{
Duplication of the right renal vein in a southern tiger cat \\ (Leopardus guttulus): a case report
}

\author{
Anieli Vidal Stocco ${ }^{1}$, Stephanie Cardoso Silva ${ }^{1}$, Karen dos Santos Toledo ${ }^{1}$, Carlos Augusto dos Santos-Sousa ${ }^{1,2}$ \\ Raquel Batista Junger de Carvalho ${ }^{3}$, Marcelo Abidu-Figueiredo ${ }^{1 *}$ \\ ${ }^{1}$ Universidade Federal Rural do Rio de Janeiro (UFRRJ), Seropédica, RJ, Brasil \\ 2 Universidade Federal do Acre (UFAC), Rio Branco, AC, Brasil \\ 3 Parque Nacional da Serra dos Órgãos, Teresópolis, RJ, Brasil
}

\section{Resumo}

0 gato-do-mato-pequeno pertence ao gênero Leopardus, que possui distribuição desde o sul dos Estados Unidos até o Chile. A redução e fragmentação de habitats de felinos ocasionaram a vulnerabilidade da espécie em estudo, segundo a Lista Vermelha de Espécies Ameaçadas. As veias renais direita e esquerda drenam seu conteúdo para a veia cava caudal. Variações anatômicas nas veias renais foram relatadas em várias espécies, como o cão, gato doméstico e humano. 0 objetivo deste artigo é relatar o aparecimento de variação numérica nas veias renais em Leopardus guttulus, com enfoque nas possibilidades de implicações clínico-cirúrgicas como anastomoses cirúrgicas, estudos imaginológicos, nefrectomias e planejamento pré-operatório para redução de riscos e complicações, como hemorragia. 0 animal foi devidamente formolizado no Laboratório de Ensino e Pesquisa em Morfologia de Animais Domésticos e Selvagens, posteriormente injetado látex colorido e dissecado. Foi observada variação numérica na veia renal no rim direito de um macho. Observou-se no rim esquerdo apenas uma veia renal medindo $0,98 \mathrm{~cm}$ de comprimento. 0 rim direito apresentou duas veias renais, sendo a primeira a fusão de duas veias interlobares em uma veia renal crânio-dorsal com 0,95 cm de comprimento e a segunda apresentando $0,98 \mathrm{~cm}$ de comprimento.

Palavras-chave: Felino. Rim. Variação anatômica.

\begin{abstract}
The southern tiger cat of the genus Leopardus is distributed from the south of the United States of America to Chile. The reduction and fragmentation of the feline's habitats has resulted in vulnerability of the species according to the Red List of Threatened Species. The right and the left renal veins empty their contents into the caudal vena cava. Anatomical variations in the renal veins have been described in several
\end{abstract}


species, like the domestic cat and human. The aim of this article was to report the appearance of variations in the number of renal veins in Leopardus guttulus, discussing the possibilities of clinical-surgical implications such as surgical anastomoses, imaging studies, nephrectomies and preoperative planning to reduce risks and complications such as hemorrhage. The animal was fixed with formalin solution at the Laboratório de Ensino e Pesquisa em Morfologia de Animais Domésticos e Selvagens, injected with colored latex and dissected. We observed a numerical variation of the renal vein in the right kidney in one male. In the left kidney, only one renal vein measuring $0.98 \mathrm{~cm}$ in length was observed. The right kidney had two renal veins, the first craniodorsal measuring $0.95 \mathrm{~cm}$ and the second caudoventral, measuring $0.98 \mathrm{~cm}$ in length.

Keywords: Feline. Kidney. Anatomical variation.

\section{Introdução}

A família Felidae, composta por 12 gêneros e 36 espécies, possui, em grande maioria, hábitos solitários e noturnos, além de necessitar de grandes áreas para a sua sobrevivência (Reis et al., 2006; Gregorini, 2014).

Devido à migração de ancestrais norteamericanos pelo Istmo do Panamá, cerca de 2-3 milhões de anos atrás (Berta, 1983; Hunt, 1989; Prevosti, 2006), atualmente há três linhagens filogenéticas ou clados na Região Neotropical, que compreende o sul do México e da Flórida, a América Central e a América do Sul. Estas linhagens podem ser diferenciadas molecular e morfologicamente em: Leopardus Gray, 1842; Puma Jardice, 1834 e Herpailurus Severtzov, 1858, sendo este muitas vezes considerado sinônimo de Puma; e Panthera Oken, 1916 (Collier e O’Brien, 1985; Jonhson e O'Brien, 1997; Jonhson et al., 2006), sendo o gênero Leopardus encontrado desde o sul dos Estados Unidos até o Chile (Nowell e Jackson, 1996; Sunquist e Sunquist, 2009).

De pequeno porte como as demais espécies do gênero, o Leopardus guttulus apresenta coloração de fundo pardo amarelado escuro, pelagem mais clara e com rosetas pequenas nas laterais do corpo, ventre branco ou cinza claro, cauda anelada e longa, e orelhas arredondadas. Como a maioria dos felídeos, a espécie enfrenta um risco elevado de extinção na natureza, sendo classificada como "vulnerável", possivelmente pela redução e fragmentação de habitat (Nascimento, 2010; Oliveira et al., 2016).

Variações numéricas das veias renais foram caracterizadas no homem (Duques et al., 2002), no cão (Reis e Tepe, 1956) e no gato (Campos et al., 2014; Stocco et al., 2014).

Segundo Satyapal et al. (1995), no homem a incidência de veias renais múltiplas é dez vezes maior no antímero direito (26\%) do que no esquerdo $(2,6 \%)$. Entretanto, Soares et al. (2014), analisando 50 amostras, dentre elas blocos abdominais e cadáveres, não observaram diferença percentual na incidência das veias renais entre os antímeros.

Nos animais mamíferos domésticos, tanto a veia renal direita quanto a esquerda drenam seu conteúdo para a veia cava caudal (König e Liebich, 2016). No entanto, o número, curso e local de drenagem das veias renais são, por vezes, motivos de muita controvérsia. Variações anatômicas das veias renais são ocasionalmente encontradas em estudos radiológicos e de rotina na dissecação da região abdominal (Santos-Sousa et al., 2016).

Santos-Sousa et al. (2016) observaram que no rim esquerdo de um coelho, a veia renal não drenava seu conteúdo para a veia cava caudal como acontece normalmente; neste caso, a veia renal esquerda não manteve nenhuma comunicação com a veia cava caudal e sofreu anastomose com a veia abdominal cranial.

Apesar do aumento na pesquisa básica e aplicada envolvendo animais silvestres, ao consultar a literatura, em particular os textos de anatomia, verifica-se que as informações referentes às veias renais desses animais, especialmente suas variações numéricas e locais de drenagem, são escassas.

Em se tratando da morfologia, há escassez de estudos na espécie, mostrando-se importante a obtenção de informações para o entendimento da anatomia do animal tanto para futuros trabalhos como para a preservação do mesmo, seja em cativeiro ou habitat natural. Neste contexto, o presente estudo visa descrever a duplicidade da veia renal em gato-do-mato-pequeno, com enfoque nas possíveis implicações clínico-cirúrgicas desta variação anatômica. 


\section{Relato de caso}

Durante as atividades de dissecção realizadas no Laboratório de Ensino e Pesquisa em Morfologia dos Animais Domésticos e Selvagens (LEPeMADS) do Departamento de Anatomia Animal e Humana, da Universidade Federal Rural do Rio de Janeiro, observou-se uma variação numérica na veia renal direita em um espécime de gato-do-mato-pequeno, macho, adulto, doado pelo Parque Nacional da Serra dos Órgãos ao LEPeMADS.

0 cadáver foi identificado e posicionado em decúbito lateral direito. A seguir, o tórax foi aberto e dissecado para evidenciação da aorta torácica, na qual foi introduzida uma sonda uretral número 6. Desse modo, o sistema arterial foi "lavado" com solução fisiológica de $\mathrm{NaCl}$ a $0,9 \%$ e, então, fixado com solução de formol a 10\%. Após, injetouse pela sonda solução aquosa (diluição 1:1) de Petrolátex S-65 (Refinaria Duque de Caxias-REDUCda Petrobrás, Duque de Caxias-RJ) misturado ao corante (Suvinil xadrez ${ }^{\circledR}$ ). Em seguida, o cadáver foi imerso em caixa de polietileno de baixa densidade, de capacidade de 50 litros, contendo solução de formol a $10 \%$ para a finalização do processo de fixação e polimerização do látex.

Decorridos sete dias da injeção do látex, procedeuse a lavagem em água corrente, abertura e dissecção da cavidade peritoneal para evidenciar os rins.

Com um paquímetro digital da marca Eda foram obtidas as medidas renais (comprimento, largura e espessura) e de comprimento das veias renais.

\section{Resultados}

O animal do sexo masculino, jovemadulto, possuía $51 \mathrm{~cm}$ de comprimento rostro-sacral, apresentando rim direito com 4,68 cm de comprimento, $2,55 \mathrm{~cm}$ de largura e 2,34 cm de espessura, localizado entre a segunda e quinta vértebras lombares (L2 e L5).

Foi observado neste rim a presença de duas veias renais: uma craniodorsal e outra caudodorsal. A primeira, próxima ao hilo renal, era formada pela confluência de duas veias interlobares, sendo uma dorsal, medindo $0,94 \mathrm{~cm}$ de comprimento, e uma ventral com $0,62 \mathrm{~cm}$ de comprimento, que confluía em uma veia renal com $0,95 \mathrm{~cm}$ de comprimento. Já a segunda veia renal, caudoventral, possuía $0,98 \mathrm{~cm}$ de comprimento (Figura1).

O rim esquerdo possuía $4,47 \mathrm{~cm}$ de comprimento, $2,73 \mathrm{~cm}$ de largura e 2,59 $\mathrm{cm}$ de espessura, estava localizado entre a segunda e quinta vértebras lombares (L2 e L5), e apresentou uma única veia renal medindo $0,98 \mathrm{~cm}$ comprimento.

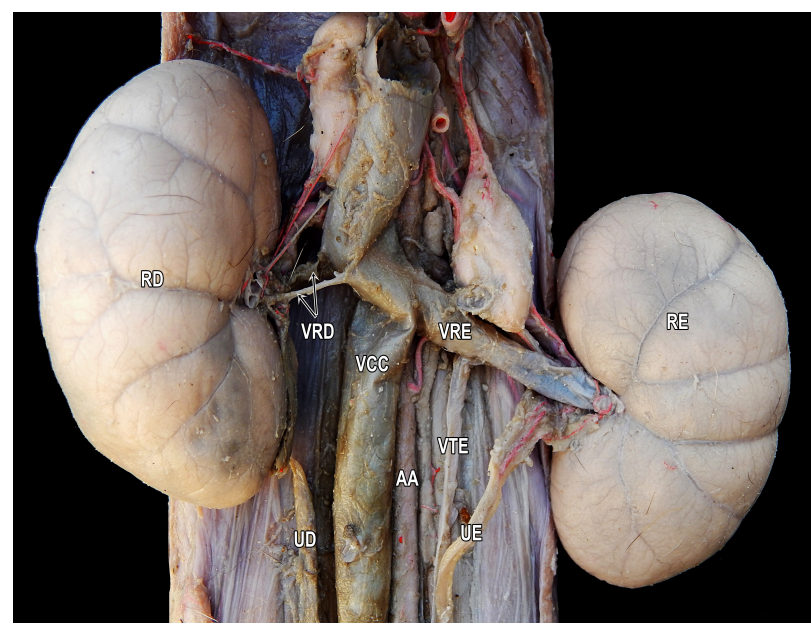

Nota: $\mathrm{RD}=$ Rim direito. $\mathrm{RE}=$ Rim esquerdo. $\mathrm{VRD}=$ veias renais direitas. $\mathrm{VRE}=$ veia renal esquerda. $\mathrm{VCC}=$ veia cava caudal. $\mathrm{UD}=$ ureter direito. $\mathrm{UE}=$ ureter esquerdo.VT E= veia testicular esquerda.

Figura 1 - Fotomacrografia da vista ventral mostrando duplicidade das veias renais direitas.

\section{Discussão}

Em estudos realizados em cadáveres humanos de homens e mulheres, Duques et al. (2002) observaram que a veia renal esquerda em $8,9 \%(n=3)$ era dupla, enquanto que em $91,1 \%(n=31)$ dos casos era única, semelhante ao observado por Bouzouita et al. (2015), que encontraram veia renal única em $88 \%$ dos casos $(n=63)$ e dupla em $11 \%(n=8)$ [cinco rins direitos e três esquerdos].

Hassan et al. (2017) encontraram em sete cadáveres humanos mestiços (11\%) variações renais diversas, dentre as quais: no antímero direito, uma veia renal tripla, uma veia renal quádrupla e uma veia renal dupla; e no antímero esquerdo, uma veia renal dupla e uma tripla. 
Variações numéricas das artérias renais foram descritas em cães (Oliveira e Guimarães, 2007; Alonso e Abidu-Figueiredo, 2008), gatos (Pestana et al., 2011) e coelhos (Almeida et al., 2013), sendo estes relatos mais frequentes em relação aos observados nas veias renais, embora Campos et al. (2014) e Stocco et al. (2014) tenham relatado veias renais duplas em ambos os rins e triplas apenas nos rins direitos de gatos.

Ao dissecarem as veias renais de 500 cães mestiços, 287 machos e 213 fêmeas, Reis e Tepe (1956) observaram a presença de veia renal direita dupla em $1 \%(n=5)$ e a veia renal esquerda se apresentou única em todos os casos, diferindo dos resultados encontrados por Fagundes et al. (1990), cuja duplicidade foi encontrada apenas no rim direito.

A complexidade das variações vasculares pode modificar as possibilidades técnicas do procedimento cirúrgico e, segundo Khamanarong et al. (2004) e Uzmansel et al. (2014), o perfeito entendimento da anatomia vascular renal proporciona eficiência e segurança nos procedimentos cirúrgicos e radiológicos. Portanto, qualquer cirurgia abdominal que necessite de mobilização ou controle hemostático dos vasos renais requer uma busca sistemática por eventuais variações anatômicas vasculares.

A divulgação dos achados das variações anatômicas é importante para a prática médica veterinária envolvendo animais domésticos $\mathrm{e}$ selvagens e deve ser realizada como forma de conscientização dos profissionais, contribuindo para o sucesso e aperfeiçoamento de diferentes protocolos clínico-cirúrgicos.

0 transplante renal pode ser uma alternativa terapêutica em gatos com estágio final de insuficiência renal. Neste caso, Bouma et al. (2003) reforçam que os requisitos para a anatomia vascular dos rins coletados são específicos para garantir o sucesso da cirurgia. Se houver uma única artéria renal, com um comprimento prévio de ramificação de pelo menos $0,5 \mathrm{~cm}$, a anastomose arterial é mais bem-sucedida. Se o pedículo vascular tem mais de uma veia, a veia com o maior comprimento e de maior diâmetro está reservada para a anastomose.

Por conseguinte, devem-se considerar variações numéricas das veias renais na execução dos procedimentos cirúrgicos, radiológicos e experimentais a fim de evitar que erros sejam cometidos por ausência de conhecimento da possibilidade de existência de veias renais duplas, ou mesmo triplas, tanto em animais domésticos como em selvagens.

\section{Referências}

Alonso LS, Abidu-Figueiredo M. Artéria renal dupla originando da aorta em cão: relato de caso. Semina Cienc Agrar. 2008;29(1):185-8.

Almeida BB, Araújo-Barreto UH, Costa OM, AbiduFigueiredo M. Double renal artery in rabbits. Biosci J. 2013; 29(5):1295-9.

Berta A. A new species of small cat (Felidae) from the Late Pliocene - Early Pleoistocene (Uquian) of Argentina. J Mammal. 1983;64(4):720-25.

Bouma JL, Aronson LR, Keith DG, Saunders HM. Use of computed tomography renal angiography for screening feline renal transplant donors. Vet Radiol Ultrasound. 2003;44(6):636-41.

Bouzouita A, Kerkeni W, Bouchiba N, Allouche M, Mighri MM, Hamdoun M, et al. Anatomical variations of renal venous vascularisation. A study of 71 three-dimensional kidney endocasts. Tunis Med. 2015;93(1):16-20.

Campos CBA, Rocha PS, Abidu-Figueiredo M. Veia renal dupla em gatos: relato de casos. Rev Acad Cienc Agrar Ambient. 2014;12(2):127-31.

Collier GE, O'Brien SJ. A molecular phylogeny of the Felidae: immunological distance. Evolution. 1985;39(3):473-87.

Duques P, Rodrigues JR, Silva Neto FB, Neto EMVS, Tolêdo ES. Estudo anatômico da veia renal esquerda de cadáveres humanos brasileiros. Medicina. 2002;35(2):184-91.

Fagundes GM, Souza A, Borelli V, Riella ACM. Contribuição ao estudo da drenagem sanguínea do rim de cães (Canis familiaris - Linnaeus, 1758). Biotemas. 1990;3(2): 117-27.

Gregorini MZ. Efeitos da perda e fragmentação de habitat sobre felinos: ecologia e genética de paisagem 
como ferramentas para a conservação [tese]. Goiás: Universidade Federal de Goiás; 2014.

Hassan SS, El-Shaarawy EA, Johnson JC, Youakim MF, Ettarh R. Incidence of variations in human cadaveric renal vessels. Folia Morphol (Warsz). 2017;76(3):394-407.

Hunt RM Jr. Biogeography of the Order Carnivora. In: Gittleman JL (Ed). Carnivore, Behavior, Ecology and Evolution. New York: Cornell University Press; 1989. p. 485-541.

Jonhson WE, O'Brien SJ. Phylogenetic reconstruction of the Felidae using 16S rRNA and NADH-5 mitochondrial genes. J Mol Evol. 1997;44(Suppl 1):S98-116.

Jonhson WE, Eizirik E, Pecon-Slattery J, Murphy WJ, Antunes A, Teeling E, et al. The late Miocene radiation of modern Felidae: a genetic assessment. Science. 2006;311(5757):73-7.

Khamanarong K, Prachaney P, Utraravichien A, Tong-Un T, Sripaoraya K. Anatomy of renal arterial supply. Clin Anat. 2004;17(4):334-6.

König HE, Liebich HG. Anatomia dos animais domésticos: texto e atlas colorido. 6 ed. Porto Alegre: Artmed; 2016. $824 \mathrm{p}$.

Nascimento FO. Revisão taxonômica do genêro Leopardus Gray, 1842 (Carnivora, Felidae) [tese]. São Paulo: Universidade de São Paulo; 2010.

Nowell K, Jackson P. Wild Cats: Status Survey and Conservation Action Plan. Glan, Suíça: IUCN, The World Conservation Union; 1996. 382 p.

Oliveira FS, Guimarães GC. Duplicidade da artéria renal em cão. Cienc Rural. 2007;37(6):1817-9.

Oliveira T, Trigo T, Tortato M, Paviolo A, Bianchi R, LeitePitman MRP. Leopardus guttulus. The IUCN Red List of Threatened Species. 2016: e.T54010476A54010576.

Pestana FM, Roza MS, Hernandez JMF, Silva BX, AbiduFigueiredo M. Artéria renal dupla em gato: relato de caso. Semina Cienc Agrar. 2011;32(1):327-32.
Prevosti FJ. New material of Pleistocene cats (Carnivore, Felidae) from Southern South America, with comments on biogeography and fossil record. Geobios. 2006;39(5):679-94.

Reis NR, Peracchi AL, Pedro WA, Lima IP (Eds). Mamíferos do Brasil. Londrina: Universidade Estadual de Londrina; 2006; p. 386-7.

Reis RH, Tepe P. Variations in the pattern of renal vessels and their relation to the type posterior vena cava in the dog (Canis Familiaris). Am J Anat. 1956;99(1):1-15.

Santos-Sousa CA, Stocco AV, Ferreira LT, Silva SC, Gomes MS, Abidu-Figueiredo M. Drenagem atípica da veia renal em coelho - Relato de caso. Rev Bras Med Vet. 2016;38(2):105-7.

Soares TRS, Dartibale C, Ferraz J, Oliveira I. Prevalência de veias renais adicionais humanas. Arq Mudi. 2014;18(1):18-27.

Stocco AV, Stocco NV, Santos-Sousa CA, Abidu-Figueiredo M. Veia renal tripla em gato: relato de casos. Rev Port Cienc Vet. 2014;109(591-592):120-2.

Satyapal KS, Rambiritch V, Pillai G. Additional renal vein: incidence and morphometry. Clin Anat. 1995;8(1):51-5.

Sunquist ME, Sunquist FC. Family Felidae (Cats). In Wilson DE, Mittermeier RA (Eds). Handbook of the Mammals of the World - Volume 1: Carnivores. Barcelona: Lynx Ediciones; 2009. p. 54-169

Uzmansel D, Ozturk NC, Kara A, Ozturk H. A rare combination of vascular variations of both kidneys. Surg Radiol Anat. 2014;36(2):195-8. 